\title{
Super-resolution on the Sphere using Convex Optimization
}

\author{
Tamir Bendory, Shai Dekel, and Arie Feuer ,Fellow, IEEE \\ Revised: October 2014, January 2015
}

\begin{abstract}
This paper considers the problem of recovering an ensemble of Diracs on a sphere from its low resolution measurements. The Diracs can be located at any location on the sphere, not necessarily on a grid. We show that under a separation condition, one can recover the ensemble with high precision by a three-stage algorithm, which consists of solving a semi-definite program, root finding and least-square fitting. The algorithm's computation time depends solely on the number of measurements, and not on the required solution accuracy. We also show that in the special case of non-negative ensembles, a sparsity condition is sufficient for recovery. Furthermore, in the discrete setting, we estimate the recovery error in the presence of noise as a function of the noise level and the super-resolution factor.
\end{abstract}

\section{INTRODUCTION}

In many cases, signals are observed on spherical manifolds. Typical examples are astrophysics (e.g. [4], [31]), gravity fields sensing [30] and geophysics [48]. A further example is spherical microphone arrays, used for spatial beam forming [37], sound recording [38] and acoustic source localization [27].

Spherical harmonics are a key tool for the analysis of signals on the sphere. For instance, the spherical microphone array was analyzed in terms of spherical harmonics in [42]. Additionally, spherical harmonics have been extensively used for various applications in computer graphics, such as modeling of volumetric scattering effects, bidirectional reflectance distribution function, and atmospheric scattering (for more graphical applications, see [50] and the references therein). Spherical harmonics are also used in medical imaging [21], [52], [55], optical tomography [2], wireless channel modeling [40] and several applications in physics such as solving potential problem in electrostatics [34], and the central potential Schrodinger equation in quantum mechanics [17]. Based on spherical harmonics analysis, new sampling theorems on the sphere for band-limited signals [6], [36] and for signals with finite rate of innovation [20] were suggested, and advanced analysis methods on the sphere were applied [29], [33]. 
Let $\mathcal{H}_{n}\left(\mathbb{S}^{d-1}\right)$ denote the space of homogeneous spherical harmonics of degree $n$, which is the restriction to the $(d-1)$ unit sphere of the homogeneous harmonic polynomials of degree $n$ in $\mathbb{R}^{d}[3]$. Each subspace $\mathcal{H}_{n}\left(\mathbb{S}^{d-1}\right)$ is of dimension

$$
a_{n, d}:=\frac{(2 n+d-2)(n+d-3) !}{n !(d-2) !}, \quad n \in \mathbb{N}, d \geq 2 .
$$

Let us denote by $\left\{Y_{n, k}\right\}, k=1, \ldots, a_{n, d}$, an orthonormal basis of $\mathcal{H}_{n}\left(\mathbb{S}^{d-1}\right)$. The set $\left\{Y_{n, k}\right\}$ is a basis for the space of square integrable functions on $\mathbb{S}^{d-1}$. Consequently, any $f \in L_{2}\left(\mathbb{S}^{d-1}\right)$ can be expanded as

$$
f=\sum_{n=0}^{\infty} \sum_{k=1}^{a_{n, d}}\left\langle f, Y_{n, k}\right\rangle Y_{n, k} .
$$

For $d=2$ the appropriate spherical harmonic basis is simply the standard Fourier basis $\left\{e^{j n \phi}\right\}$.

In this work we focus on the two-dimensional sphere $\mathbb{S}^{2}$ embedded in $\mathbb{R}^{3}$. In this case, any point on the sphere is parametrized by $\xi:=(\phi, \theta) \in[0,2 \pi) \times[0, \pi]$. The appropriate orthonormal spherical harmonics basis is given by ${ }_{1}^{1}$

$$
Y_{n, k}(\xi)=A_{n, k} e^{j k \phi} P_{n, k}(\cos \theta), \quad 0 \leq n<\infty, \quad-n \leq k \leq n,
$$

where $P_{n, k}(x)$ is an associated Legendre polynomial of degree $n$ and order $k$, and $A_{n, k}$ is a normalization factor, given by

$$
A_{n, k}:=\sqrt{\frac{2 n+1}{4 \pi} \frac{(n-|k|) !}{(n+|k|) !}} .
$$

The distance on the sphere between any two points $\xi_{i}, \xi_{j} \in \mathbb{S}^{2}$ is given by

$$
d\left(\xi_{i}, \xi_{j}\right)=\arccos \left(\xi_{i} \cdot \xi_{j}\right) .
$$

Consider a Dirac ensemble on the bivariate sphere $\mathbb{S}^{2}$

$$
f=\sum_{m} c_{m} \delta_{\xi_{m}}
$$

where $\delta_{\xi}$ is a Dirac measure, $\left\{c_{m}\right\}$ are real weights, and $\Xi:=\left\{\xi_{m}\right\} \subset \mathbb{S}^{2}$ are distinct locations on the sphere, namely the signal support. Let us denote by $V_{N}$ the space of spherical harmonics of degree $\leq N$. We assume that the only information we have on the signal $f$ is its 'orthogonal projection' onto $V_{N}$, i.e.

$$
y_{n, k}:=\left\langle f, Y_{n, k}\right\rangle=\sum_{m} c_{m} \bar{Y}_{n, k}\left(\xi_{m}\right), \quad 0 \leq n \leq N, \quad-n \leq k \leq n .
$$

In matrix notations, [I.4) is presented as

$$
y=F_{N} f
$$

${ }^{1}$ Note that $k$ has a different range here than in $\mathrm{I} .1$. 
where $F_{N}$ is a semi-infinite matrix with $(N+1)^{2}$ rows and $y$ is a column stacked vector of $\left\{y_{n, k}\right\}$. That is to say, $F_{N}$ is a projection operator onto $V_{N}$. The adjoint operator is denoted as $F_{N}^{*}$. Our first main contribution (see Theorem II.2) is an algorithm that recovers exactly the underlying signal from its projection onto $V_{N}$.

To be clear, we assume that the high spherical harmonic coefficients are annihilated before any sampling procedure occurs. In the spatial domain, the projection onto $V_{N}$ can be computed by approximately $2(N+1)^{2}$ samples based on a stable equiangular sampling scheme on the sphere [36]. A recent work derives an accurate computation of the projection using only $(N+1)^{2}$ samples [28].

As a special case of the analog model, we also define a discrete configuration where the signal is known to lie on a grid. Consider a discrete signal on the sphere

$$
f=\sum_{m} c_{m} \delta_{\xi_{m}}, \quad \xi_{m} \in \Xi \subset \mathbb{S}_{L}^{2}
$$

where $\mathbb{S}_{L}^{2}$ is a predefined grid, not necessarily uniform. We assume that any pair of points on the grid $\xi_{i}, \xi_{j} \in \mathbb{S}_{L}^{2}$ obey $d\left(\xi_{i}, \xi_{j}\right) \geq 1 / L$ for some $L \geq 1 / \pi$. This measurements model is equivalent to

$$
y=F_{N}^{L} f,
$$

where $F_{N}^{L}$ is the spherical harmonics matrix. This model will serve as the basis for our main result on recovery in noisy setting (see Theorem [II.3]. For the discrete model, we define the notion of super-resolution factor (SRF) (see also [14]). $\mathrm{SRF}$ is defined as

$$
S R F:=\frac{L}{N},
$$

and represents the ratio between the desired and the measured resolutions. This agrees with the analog model $([.3)$ when $S R F \rightarrow \infty$.

Our model reflects the fact that sensing systems have a physical limit, determining the highest resolution the system can achieve. In these cases, the observer has access solely to a coarse scale measurements of the underlying signal. The problem of recovering the fine details of a signal from its low-resolution measurements can be interpreted as super-resolution on the sphere problem.

This work was inspired by the seminal paper of Candes and Fernandez-Granada [14], who investigated the recovery of Dirac ensemble on the interval $[-\pi, \pi]$ from its low $2 N+1$ Fourier coefficients. The main result of this paper states that if the Diracs are separated by at least $\frac{4 \pi}{N}$, the signal can be recovered as the unique solution of a tractable convex optimization problem. This result holds for higher dimensions as well under a separation condition of $C_{d} / N$, where $C_{d}$ is a constant which depends only on the dimension of the problem (e.g. $\left.C_{1}=4 \pi\right)$. A consecutive paper [13] showed that the recovery is robust to noisy measurements. Similar results are given for support detection from low Fourier coefficients [5], 
[24], recovery of non-uniform splines from their projection onto spaces of algebraic polynomials [9], [19] and recovery of streams of pulses [7], [10]. (see also [18]).

The configuration in (I.5) resembles the formulation in compressed sensing (CS) (e.g. [15], [22]). Using CS methods, the authors of [44] have suggested to recover a s-sparse signal with bandwidth $N$ (in the sperical harmonics domain) by only $m \sim s N^{1 / 2} \log ^{4}\left(N^{2}\right)$ samples using $\ell_{1}$ minimization. In [12], the number of the required samples was reduced to $m \sim s N^{1 / 3} \log ^{4}\left(N^{2}\right)$. However, we note that there exist two important distinctions between the framework suggested here and CS. Firstly, CS usually works on discrete signals, while $(I .3)$ describes an analog model, namely the support $\Xi$ can comprise any point on the sphere. Secondly, CS sampling matrix is required to be incoherent in some sense, which typically leads to random sampling strategies, while in [I.4 the measurements consist of the low-end of the spherical harmonics representation, and as a result are extremely coherent.

Recently, a number of works suggest to super-resolve signals by a semi-definite program [11], [14], [16], [53], [54], [56]. We extend this line of work to signals on a sphere. The first result of this paper is that Algorithm 1 recovers a signal of the form $[I .3$ from its low-resolution measurements (I.4) using a three-stage algorithm consists of solving a semi-definite program, root findind on the sphere, and least square fitting. This holds provided that the Diracs are separated by at least $\frac{\nu}{N}$ for some numerical constant $\nu$.

In [20], the authors suggest a parametric method ('finite rate of innovation' type) to reconstruct exactly a stream of $\mathrm{K}$ Diracs on the sphere from $3 K$ samples, which is optimal (that is to say, the number of samples is equal to the number of degrees of freedom). This approach assumes a known number of Diracs, but does not assume any separation between the Diracs. Generally, parametric methods such as MUSIC, matrix pencil and ESPRIT [26], [45], [47], [51] tend to be unstable in the presence of noise or model mismatch. Our second result generalizes [14] to the sphere in the discrete setting [I.6] and provides an estimate of the recovery error in the presence of noise or model mismatch.

The rest of the paper is organized as follows. Section $\Pi$ presents the two main results of this paper, and sections $\Pi$ and $\mathrm{IV}$ prove them. Section $\mathrm{V}$ is devoted to experimental results. Section $\mathrm{VI}$ elaborates on the necessity of the separation condition and ultimately Section VII concludes the paper and relates it to an ongoing research.

\section{MAIN RESUlTS}

In a previous paper [8], the authors established a sufficient condition for exact recovery of a signal of the form of (I.3) from its projection onto $V_{N}$ using a convex optimization method. The recovery relies on the following separation condition:

Definition II.1. A set of points $\Xi \subset \mathbb{S}^{2}$ is said to satisfy the minimal separation condition for (sufficiently large) $N$ if

$$
\Delta:=\min _{\xi_{i}, \xi_{j} \in \Xi, \xi_{i} \neq \xi_{j}} d\left(\xi_{i}, \xi_{j}\right) \geq \frac{\nu}{N}
$$


where $\nu$ is a fixed constant that does not depend on $N$.

Under the separation condition, the points $\xi_{m} \in \Xi$ are center of pairwise disjoint caps of area $2 \pi\left(1-\cos \left(\frac{\nu}{2 N}\right)\right)[3]$. Consequently, the number of points on the sphere can be roughly estimated by $\frac{2}{1-\cos \left(\frac{\nu}{2 N}\right)}$ (for a tighter estimation, see $[43]$ ). In an noise-free environment, a separation constant of $2 \pi$ seems to ensure exact recovery (see Figure 2). This separation coincides with the spatial resolution of the projection of $f$ onto $V_{N}$, namely $F_{N}^{*} F_{N} f:=P_{N} f[41]$. In a noisy environment, we increased the separation constant to be $\nu=2.5 \pi$.

Before presenting the main theorem, we introduce the notion of half space. A half space is a set $\mathcal{H} \subset \mathbb{Z}^{d}$, satisfying $\mathcal{H} \cap(-\mathcal{H})=\{0\}, \mathcal{H} \cup(-\mathcal{H})=\mathbb{Z}^{d}$, and $\mathcal{H}+\mathcal{H} \subset \mathcal{H}[23$. Figure 1 demonstrates the two half spaces in two dimensions.

We make use of the following notations. Let $\Theta_{k}$ be an elementary Toeplitz matrix with ones on the $k$ diagonal and zeros elsewhere (the main diagonal is indexed by zero), let $\otimes$ be a Kronecker product and $\Theta_{k, \ell}:=\Theta_{\ell} \otimes \Theta_{k}$. tr $(X)$ denotes the trace of the matrix $X$, and $\delta_{k, \ell}$ denotes a Kronecker Delta function, defined as

$$
\delta_{k, \ell}= \begin{cases}1 & k=\ell=0 \\ 0 & \text { othewise }\end{cases}
$$
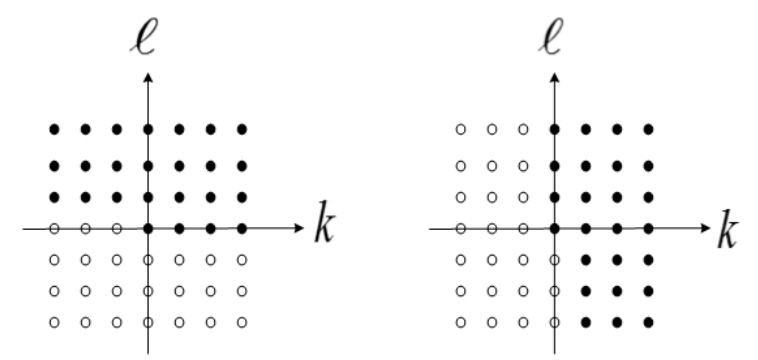

Fig. 1: Half spaces in two dimensions.

Algorithm 1 consists of three stages: solving a semi-definite program, root finding and least-square fitting. Although the model (I.3) reflects an analog (infinite dimensional) signal, we suggest to recover it from (I.4) by a semi-definite program with $\mathcal{O}\left(N^{4}\right)$ variables. This results in an unconstrained accuracy and no dependence on any discretization step.

Theorem II.2. Let $\Xi=\left\{\xi_{m}\right\}$ be the support of a signed measure $f$ of the form $I .3$. Let $\left\{Y_{n, k}\right\}_{n=0}^{N}$ be any spherical harmonics basis for $V_{N}\left(\mathbb{S}^{2}\right)$ and let $y_{n, k}=\left\langle f, Y_{n, k}\right\rangle, 0 \leq n \leq N,-n \leq k \leq n$. If $\Xi$ satisfies the separation condition of Definition II.1, then Algorithm 1 recovers $f$ exactly with $\varepsilon=0$ in (II.2). Furthermore, Algorithm 1 recovers a non-negative signal (i.e. $c_{m}>0$ ) exactly as long as $f$ has at most $N$ non-zero values. 


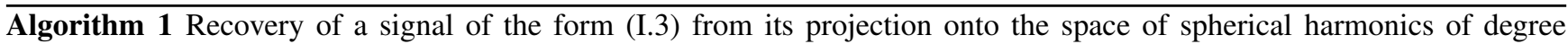
$\leq N$.

Input: The signal's projection onto $V_{N}$ (I.4).

Output: A signal of the form (I.3).

1) Solve the semi-definite program

$$
\max _{\alpha, Q}\langle y, \alpha\rangle-\varepsilon\|\alpha\|_{2} \quad \text { subject to }\left[\begin{array}{cc}
Q & \grave{h} \\
\grave{h}^{*} & 1
\end{array}\right] \succeq 0, \quad \operatorname{tr}\left(\Theta_{k, \ell} Q\right)=\delta_{k, \ell}, \quad(k, \ell) \in \mathcal{H},
$$

where $Q \in \mathbb{C}^{(2 N+1)^{2} \times(2 N+1)^{2}}$ is a Hermitian matrix, $\grave{h} \in \mathbb{C}^{(2 N+1)^{2}}$ is related to $\alpha$ through $($ III.5), and $\mathcal{H}$ is a half space.

2) Define $q(\xi)=F_{N}^{*} \alpha(\xi)$, and find the roots of the polynomials $1-q(\xi)$ and $1+q(\xi)$. These roots are denoted as $\hat{\Xi}:=\left\{\hat{\xi}_{m}\right\}$.

3) Solve the least-square system

$$
\sum_{\hat{\xi}_{m} \in \hat{\Xi}} \hat{c}_{m} \bar{Y}_{n, k}\left(\hat{\xi}_{m}\right)=y_{n, k}, \quad n=0, \ldots, N, k=-n, \ldots, n .
$$

4) Construct the recovered signal as

$$
\hat{f}=\sum_{m} \hat{c}_{m} \delta_{\hat{\xi}_{m}}
$$

In addressing the noisy case, we consider the following discrete model. Let us denote by $D_{L}$ the set of measures of the form I.6, that is, $f=\sum_{m} c_{m} \delta_{\xi_{m}}$, with $\xi_{m} \in \mathbb{S}_{L}^{2}, \forall m$. Observe that $f$ can be regarded as a discrete signal $\left\{c_{m}\right\}$ indexed by the set $\mathbb{S}_{L}^{2}$. Therefore, we may also define for $f \in D_{L},\|f\|_{\ell_{p}}:=\left(\sum_{m}\left|c_{m}\right|^{p}\right)^{1 / p}, p \geq 1$. Note that for $f \in D_{L}$, $\|f\|_{T V}=\|f\|_{\ell_{1}}$ (see Section III for definition of the TV norm for measures). Next, we consider noisy input data of the type

$$
y_{n, k}:=\left\langle f, Y_{n, k}\right\rangle+\eta_{n, k}=\sum_{m} c_{m} \bar{Y}_{n, k}\left(\xi_{m}\right)+\eta_{n, k}, \quad 0 \leq n \leq N, \quad-n \leq k \leq n,
$$

where $f \in D_{L}$, where $\eta_{n, k}$ is an additive noise.

The following result shows that the recovery error using $\ell_{1}$ minimization is proportional to $S R F^{2}$ and the noise level.

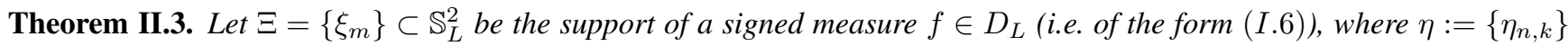
satisfies $\|\eta\|_{2} \leq \varepsilon$. Let $\left\{Y_{n, k}\right\}_{n=0}^{N}$ be any spherical harmonics orthobasis for $V_{N}\left(\mathbb{S}^{2}\right)$ and let $\left\{y_{n, k}\right\}$ be as in $(I I .3)$. For 
sufficiently large L, if $\Xi$ satisfies the separation condition of Definition II.1 then the solution $\hat{f}$ of

$$
\min _{g \in D_{L}}\|g\|_{\ell_{1}} \quad \text { subject to } \quad\left\|y-F_{N} g\right\|_{\ell_{2}} \leq \varepsilon
$$

satisfies

$$
\|\hat{f}-f\|_{\ell_{1}} \leq C_{0} S R F^{2} \varepsilon,
$$

where $C_{0}$ is a numerical constant.

We have chosen to work with a bounded noise, however our technique can be extended to other noise models. For instance, suppose that $\eta_{n, k}$ are iid entries $\mathcal{N} \sim\left(0, \sigma^{2}\right)$. In this case we obtain the following corollary:

Corollary II.4. Consider the model $[I I .3)$ and suppose that $\eta_{n, k}$ are iid entries $\mathcal{N} \sim\left(0, \sigma^{2}\right)$. Fix $\gamma>0$. For sufficiently large L, if $\Xi$ satisfies the separation condition of Definition II.1 then the solution $\hat{f}$ of II.4 with $\varepsilon=(N+1)(1+\gamma) \sigma$ satisfies

$$
\|\hat{f}-f\|_{\ell_{1}} \leq C_{0}(1+N)(1+\gamma) S R F^{2} \sigma,
$$

with probability of at least $1-e^{-\frac{1}{2}(N+1)^{2} \gamma^{2}}$, where $C_{0}$ is a numerical constant.

Theorem II.3 and Corollary II.4 are proved in Section IV.

\section{Proof of Theorem}

The proof of Theorem $\Pi 1.2$ relies on a few results from [8]. To this end, recall the following definition [8], [46]:

Definition III.1. Let $\mathcal{B}(A)$ be the Borel $\sigma$-Algebra on a compact space $A$, and denote by $\mathcal{M}(A)$ the associated space of real Borel measures. The Total Variation of a real Borel measure $v \in \mathcal{M}(A)$ over a set $B \in \mathcal{B}(A)$ is defined by

$$
|v|(B)=\sup \sum_{k}\left|v\left(B_{k}\right)\right|
$$

where the supremum is taken over all partitions of $B$ into a finite number of disjoint measurable subsets. The total variation $|v|$ is a non-negative measure on $\mathcal{B}(A)$, and the Total Variation (TV) norm of $v$ is defined as

$$
\|v\|_{T V}=|v|(A)
$$

In short, the total variation norm of a signed measure can be interpreted as the generalization of $\ell_{1}$ norm to the real line. This is not the total variation of a function, a frequently-used regularizer in signal processing (see [35] for the definition of the discrete total variation on the sphere). For a measure of the form of (II.3), it is easy to see that

$$
\|f\|_{T V}=\sum_{m}\left|c_{m}\right| .
$$


The following lemma concerns the existence of an interpolating polynomial as follows [8]:

Lemma III.2. If $\Xi$ satisfies the separation condition of Definition II.1 then there exists a polynomial $q \in V_{N}$ such that

$$
\begin{aligned}
q\left(\xi_{m}\right) & =u_{m}, \quad \forall \xi_{m} \in \Xi \\
q(\xi) & <1, \quad \xi \notin \Xi
\end{aligned}
$$

for any signed set $\left\{u_{m}\right\}$ with $\left|u_{m}\right|=1$.

The main Theorem of [8] is the following:

Theorem III.3. Let $\Xi=\left\{\xi_{m}\right\}$ be the support of a signed measure $f$ of the form $\left[I .3\right.$. Let $\left\{Y_{n, k}\right\}_{n=0}^{N}$ be any spherical harmonics basis for $V_{N}\left(\mathbb{S}^{2}\right)$ and let $y_{n, k}=\left\langle f, Y_{n, k}\right\rangle, 0 \leq n \leq N,-n \leq k \leq n$. If $\Xi$ satisfies the separation condition of Definition II.1, then $f$ is the unique solution of

$$
\min _{g \in \mathcal{M}\left(\mathbb{S}^{2}\right)}\|g\|_{T V} \quad \text { subject to } \quad F_{N} g=y
$$

where $\mathcal{M}\left(\mathbb{S}^{2}\right)$ is the space of signed Borel measures on $\mathbb{S}^{2}$.

Theorem III.3 states that if the underlying signal satisfies the separation condition of Definition II.1 then the signal is the unique solution of the TV minimization (III.1). Furthermore, in the case of non-negative signals it has been shown that the solution of [III.1) is precise as long as the signal has at most $N$ non-zero values, that is the separation condition may be replaced by a weaker sparsity condition [8].

The challenge of solving (III.1) is that the analog nature of the signal dictates an infinite-dimensional problem. One approach to alleviate this problem is to assume that the signal lies on a grid. In this case, the TV minimization reduces to standard $\ell_{1}$ minimization. However, the discretization leads to an unavoidable error, which can be mitigated by refining the grid, which in turn increases the problem complexity. This case is analyzed in Section $\mathrm{V}$ In contrast, we suggest a different approach with (theoretically) unlimited accuracy and no dependence on discretization step.

The algorithm consists of three steps. First, we reformulate the dual problem of TV minimization as a finite semi-definite programming. Later on, we use the dual solution to locate the signal's support by root finding. Finally, we estimate the amplitudes (i.e. the weights $c_{m}$ ) by least-square estimation.

With the notation $\alpha:=\left\{\alpha_{n, k}\right\}$, let $F_{N}^{*} \alpha(\xi):=\sum_{0 \leq n \leq N, k} \alpha_{n, k} Y_{n, k}(\xi)$. We assume that $\alpha$ belongs to the subspace of vectors for which

$$
\left\|F_{N}^{*} \alpha\right\|_{\infty}:=\max _{\xi \in S^{2}}\left|\sum_{n, k} \alpha_{n, k} Y_{n, k}(\xi)\right| \leq 1 .
$$


Under the separation condition, this give us the following duality

$$
\begin{aligned}
\max _{\alpha}\langle y, \alpha\rangle & :=\max _{\alpha}\left\langle F_{N} f, \alpha\right\rangle=\max _{\alpha}\left\langle f, F_{N}^{*} \alpha\right\rangle \\
& =\max _{\alpha} \int_{S^{2}} F_{N}^{*} \alpha(\xi) d f(\xi) \\
& =\int_{S^{2}} q(\xi) d f(\xi)=\|f\|_{T V},
\end{aligned}
$$

where $q$ is the polynomial from Lemma III.2. Therefore, (III.1) is translated to

$$
\max _{\alpha}\langle y, \alpha\rangle \quad \text { s.t. } \quad\left\|F_{N}^{*} \alpha\right\|_{\infty} \leq 1
$$

Observe that the objective function is finite dimensional, whereas the constraint is of infinite dimension. To this end, we state the following variant of the Bounded Real Lemma [23]:

Lemma III.4. Consider a causal trigonometric polynomial of the form

$$
H\left(\omega_{1}, \omega_{2}\right)=\sum_{k, \ell=0}^{N} h_{k, \ell} e^{-j\left(\omega_{1} k+\omega_{2} \ell\right)} .
$$

The following inequality holds

$$
\left|H\left(\omega_{1}, \omega_{\mathbf{2}}\right)\right| \leq 1, \quad \forall\left[\omega_{1}, \omega_{2}\right] \in[-\pi, \pi] \times[-\pi, \pi]
$$

if and only if there exist a Hermitian matrix $Q \succeq 0$ such that

$$
\left[\begin{array}{cc}
Q & h \\
h^{*} & 1
\end{array}\right] \succeq 0, \quad \delta_{k, \ell}=\operatorname{tr}\left(\Theta_{k, \ell} Q\right), \quad k, \ell \in \mathcal{H},
$$

where $h$ is a column stacked vector of $\left\{h_{k, \ell}\right\}$ and $\mathcal{H}$ is a half space.

Applying the Bounded Real Lemma, we can now show that the constraint of (III.2) can be recast as the intersection of a cone of semi-definite matrix with an affine hyperplane:

Lemma III.5. $\left\|F_{N}^{*} \alpha\right\|_{\infty} \leq 1$ if and only if there exists a Hermitian matrix $Q \in \mathbb{C}^{(2 N+1)^{2} \times(2 N+1)^{2}}$ such that

$$
\left[\begin{array}{cc}
Q & \grave{h} \\
\grave{h}^{*} & 1
\end{array}\right] \succeq 0, \quad \operatorname{tr}\left(\Theta_{k, \ell} Q\right)=\delta_{k, \ell}, \quad(k, \ell) \in \mathcal{H}
$$

where $\mathcal{H}$ is a half plane, $\grave{h} \in \mathbb{C}^{(2 N+1)^{2}}$ is a column stacked vector of $\grave{h}_{k, \ell}$ given by

$$
\grave{h}_{k, \ell}=\sum_{n=0}^{N} h_{n, k, \ell}, \quad h_{n, k, \ell}:= \begin{cases}A_{n, k} \alpha_{n, k} \beta_{n, k, \ell} & k, \ell \in[-n, n], \\ 0 & \text { o.w. }\end{cases}
$$


$\beta_{n, k, \ell}$ are given by the unique trigonometric decomposition of the associated Legendre polynomial of order $n$ and degree k, i.e. $P_{n, k}(\cos \theta)=\sum_{\ell=-n}^{n} \beta_{n, k, \ell} e^{j \ell \theta}$, and $A_{n, k}$ are given in $(I .2)$.

Proof: Fix a point on the two-dimensional sphere $\xi:=(\theta, \phi) \in \mathbb{S}^{2}$. A spherical harmonic polynomial of degree $N$ is of the form

$$
\begin{aligned}
F_{N}^{*} \alpha(\xi) & =\sum_{n=0}^{N} \sum_{k=-n}^{n} \alpha_{n, k} Y_{n, k}(\xi) \\
& =\sum_{n=0}^{N} \sum_{k=-n}^{n} \alpha_{n, k} A_{n, k} e^{j k \phi} P_{n, k}(\cos \theta) .
\end{aligned}
$$

$P_{n, k}(\cos \theta)$ takes the form of

$$
P_{n, k}(\cos \theta)=(\sin \theta)^{|k|} L_{n}^{(k)}(\cos \theta),
$$

where $L_{n}^{(k)}$ is the $k^{t h}$ derivative of the Legendre polynomial of degree $n$. Hence, $P_{n, k}(\cos \theta)$ is a trigonometric polynomial of degree $n$, and has an expansion $P_{n, k}(\cos \theta)=\sum_{\ell=-n}^{n} \beta_{n, k, \ell} e^{j \ell \theta}$ for unique coefficients $\left\{\beta_{n, k, \ell}\right\}_{\ell}$. Consequently, we write (III.6) as

$$
\begin{aligned}
F_{N}^{*} \alpha(\xi) & =\sum_{n=0}^{N} \sum_{k=-n}^{n} \sum_{\ell=-n}^{n} \alpha_{n, k} A_{n, k} \beta_{n, k, \ell} e^{j \ell \theta} e^{j k \phi} \\
& =\sum_{k=-N}^{N} \sum_{\ell=-N}^{N} \grave{h}_{k, \ell} e^{j \ell \theta} e^{j k \phi},
\end{aligned}
$$

where $\grave{h}_{k, \ell}$ is given in III.5. Now, The Bounded Real Lemma can be directly applied in our case, since the polynomial $e^{j N(\theta+\phi)} F_{N}^{*} \alpha(\xi)$ is causal and has the same magnitude as $F_{N}^{*} \alpha(\xi)$. This completes the proof.

Using Lemma III.5, the dual problem [III.2] is equivalent to

$$
\max _{\alpha, Q}\langle y, \alpha\rangle \quad \text { s.t. equation III.4 is satisfied. }
$$

This is a semi-definite programming optimization problem, which can be solved using off-the-shelf software. Note that there are $(2 N+2)^{4} / 2$ decision variables, without any dependence on the solution accuracy.

Define $q(\xi):=F_{N}^{*} \alpha(\xi)$, where $\alpha(\xi)$ is the solution of [III.8. Denote the roots of the polynomials $1-q(\xi)$ and $1+q(\xi)$ by $\hat{\Xi}:=\left\{\hat{\xi}_{m}\right\}$ and recall that we know that $q(\xi)$ takes the values $\operatorname{sign}\left(c_{m}\right)$ at $\xi_{m} \in \Xi$. Consequently, $\Xi \subseteq \hat{\Xi}$. Once we find the support, we can find the unknown coefficients by solving the least square system:

$$
\sum_{\hat{\xi}_{m} \in \hat{\Xi}} \hat{c}_{m} \bar{Y}_{n, k}\left(\hat{\xi}_{m}\right)=y_{n, k}, \quad n=0, \ldots, N, k=-n, \ldots, n .
$$

We note that although the detected support may be larger than the actual support, the least-square solution (III.9) will set the values of the signal to zero at these points. 
The sole situation in which our algorithm fails to recover the signal is when $q(\xi)=1$ or $q(\xi)=-1$ for all $\xi$. However, this situation will rarely occur if (III.8) is solved using standard interior point method. More precisely, according to the analysis in Section 4 of [14], $q(\xi)$ will not get a constant value if there exists a solution to [III.8) such that $|q(\xi)|<1$ for some $\xi \in S^{2}$. Indeed, in the course of our experiments this situation never occurred.

\section{Proof of Theorem $\coprod .3$ and Corollary}

Let $\hat{f} \in D_{L}$ be the solution of the optimization problem [II.4, with $\|\hat{f}\|_{T V}=\|\hat{f}\|_{\ell_{1}} \leq\|f\|_{\ell_{1}}=\|f\|_{T V}$ and let $h \in D_{L}$, $h(\xi):=\hat{f}(\xi)-f(\xi)$. We decompose $h$ as

$$
h=h_{\Xi}+h_{\Xi},
$$

where $h_{\Xi}$ and $h_{\Xi^{C}}$ are the parts of $h$ with support in $\Xi$ and $\Xi^{C}$, respectively. If $h_{\Xi}=0$, then $h=0$. Otherwise, $h_{\Xi^{C}} \neq 0$ which implies the contradiction $\|\hat{f}\|_{\ell_{1}}>\|f\|_{\ell_{1}}$. Using the notation $P_{N}:=\left(F_{N}\right)^{*} F_{N}$, we decompose the measure $h$ into 'low' and 'high' resolution parts,

$$
h_{L}=P_{N} h d \xi, \quad h_{H}=h-h_{L},
$$

where $d \xi$ is the usual surface area measure on the sphere, so that $\|h\|_{\ell_{1}}=\|h\|_{T V} \leq\left\|h_{L}\right\|_{T V}+\left\|h_{H}\right\|_{T V}$.

We commence by assuming that $\|\eta\|_{2} \leq \varepsilon$. This in turn implies that

$$
\left\|F_{N} f-y\right\|_{\ell_{2}}=\|\eta\|_{\ell_{2}} \leq \varepsilon \text {. }
$$

Using the fact that $\left\{Y_{n, k}\right\}$ is an orthobasis and then (II.4) we get

$$
\begin{aligned}
\left\|P_{N} h\right\|_{L_{2}} & =\left\|F_{N} h\right\|_{\ell_{2}} \\
& \leq\left\|y-F_{N} f\right\|_{\ell_{2}}+\left\|y-F_{N} \hat{f}\right\|_{\ell_{2}} \\
& \leq 2 \varepsilon .
\end{aligned}
$$

Consequently, we have the following estimation for the low resolution part $h_{L}$ :

$$
\left\|h_{L}\right\|_{T V}=\left\|P_{N} h\right\|_{L_{1}} \leq 2 \sqrt{\pi}\left\|P_{N} h\right\|_{L_{2}} \leq 4 \sqrt{\pi} \varepsilon .
$$

Next, we need to estimate the 'high frequency' part $h_{H}$. We denote by $h_{H, \Xi}$ and $h_{H, \Xi C}$ the parts of $h_{H}$ with support on $\Xi$ and $\Xi^{C}$, respectively. By assumption, the support of $f, \Xi:=\left\{\xi_{m}\right\}$ satisfies the separation condition of Definition II.1. Therefore, by Lemma III.2, there exists a polynomial $q \in V_{N}$ such that $q\left(\xi_{m}\right)=\operatorname{sgn}\left(h_{H}\left(\xi_{m}\right)\right)$ for all $\xi_{m} \in \Xi$ and $|q(\xi)|<1$ for all $\xi \in \mathbb{S}_{L}^{2} \backslash \Xi$. By construction,

$$
\left\langle q, h_{H}\right\rangle=\left\langle P_{N} q, h_{H}\right\rangle=\left\langle q, P_{N}\left(h-h_{L}\right)\right\rangle=0
$$


So,

$$
0=\left\langle q, h_{H}\right\rangle=\left\langle q, h_{H, \Xi}\right\rangle+\left\langle q, h_{H, \Xi C}\right\rangle \geq\left\|h_{H, \Xi}\right\|_{T V}-q_{m a x}^{C}\left\|h_{H, \Xi C}\right\|_{T V},
$$

where

$$
q_{\max }^{C}:=\max _{\xi \in \mathbb{S}_{L}^{2} \backslash \Xi}|q(\xi)|
$$

Since $\hat{f}$ has minimal $T V$ norm in $D_{L}$,

$$
\begin{aligned}
\|f\|_{T V} & \geq\|f+h\|_{T V} \geq\left\|f+h_{H}\right\|_{T V}-\left\|h_{L}\right\|_{T V} \\
& \geq\|f\|_{T V}+\left\|h_{H, \Xi C}\right\|_{T V}-\left\|h_{H, \Xi}\right\|_{T V}-\left\|h_{L}\right\|_{T V} \\
& \geq\|f\|_{T V}+\left(1-q_{\text {max }}^{C}\right)\left\|h_{H, \Xi C}\right\|_{T V}-\left\|h_{L}\right\|_{T V} .
\end{aligned}
$$

Hence,

$$
\begin{aligned}
\|h\|_{T V} & \leq\left\|h_{L}\right\|_{T V}+\left\|h_{H}\right\|_{T V} \leq\left\|h_{L}\right\|_{T V}+\left\|h_{H, \Xi}\right\|_{T V}+\left\|h_{H, \Xi C}\right\|_{T V} \\
& \leq\left\|h_{L}\right\|_{T V}+\left(1+q_{\text {max }}^{C}\right)\left\|h_{H, \Xi C}\right\|_{T V} \\
& \leq\left\|h_{L}\right\|_{T V}+\frac{\left(1+q_{\text {max }}^{C}\right)}{\left(1-q_{\text {max }}^{C}\right)}\left\|h_{L}\right\|_{T V} \\
& \leq \frac{2\left\|h_{L}\right\|_{T V}}{\left(1-q_{\text {max }}^{C}\right)} \leq \frac{8 \sqrt{\pi} \varepsilon}{\left(1-q_{\text {max }}^{C}\right)} .
\end{aligned}
$$

In order to estimate $q_{\text {max }}^{C}$, we make use of several results from [8]. Let $\xi \in \mathbb{S}_{L}^{2} \backslash \Xi$. We first handle the case where $1 / L \leq d\left(\xi, \xi_{m}\right) \leq s / N$, for some $\xi_{m} \in \Xi$, where the constant $s>0$ is determined by Lemma 4.3 in [8]. We provide an upper bound for $|q(\xi)|$, by analyzing the Taylor remainder of the univariate function $F(\theta):=q(r(\theta))$, with $r(\theta):=$ $\left(1-\theta / d\left(\xi, \xi_{m}\right)\right) \xi_{m}+\left(\theta / d\left(\xi, \xi_{m}\right)\right) \xi, 0 \leq \theta \leq d\left(\xi, \xi_{m}\right)$. By construction, $q\left(\xi_{m}\right)= \pm 1$, so without loss of generality, let us assume $F(0)=q\left(\xi_{m}\right)=1$. Also, by the construction in [8], in this case, $q$ has a local maximum at $\xi_{m}$ and so $F^{\prime}(0)=0$. Next, by Lemma 4.3 in [8] there exists an absolute constant $c>0$, such that $F^{\prime \prime}(\theta) \leq-c N^{2}$, for all $0 \leq \theta \leq s / N$. Therefore, we can apply the Taylor Remainder theorem to bound

$$
\begin{aligned}
q(\xi) & =F\left(d\left(\xi, \xi_{m}\right)\right) \\
& \leq 1-\frac{c N^{2}}{2} d\left(\xi, \xi_{m}\right)^{2} \\
& \leq 1-\frac{c N^{2}}{2} \frac{1}{L^{2}}=1-\frac{\tilde{c}}{S R F^{2}},
\end{aligned}
$$


where SRF is defined by [I.7]. We now prove the case $1 / L \leq s / N \leq d\left(\xi, \xi_{m}\right), \forall \xi_{m} \in \Xi$. By Lemma 4.4 in [8], there exists $0<\delta<s$, such that $|q(\xi)| \leq(1+\delta) /(1+s)$. Therefore, if $L$ is chosen sufficiently large, such that

$$
\left(\frac{N}{L}\right)^{2} \leq \tilde{c}^{-1} \frac{s-\delta}{s+1}
$$

then,

$$
|q(\xi)| \leq \frac{1+\delta}{1+s} \leq 1-\frac{\tilde{c}}{S R F^{2}}
$$

Applying the upper bound on $q_{\max }^{C}$ gives

$$
\|h\|_{\ell_{1}}=\|h\|_{T V} \leq \frac{8 \sqrt{\pi} \varepsilon}{\tilde{c}} S R F^{2} .
$$

This concludes the proof of Theorem III.3

In order to prove Corollary II.4 we assume that $\eta_{n, k}$ are iid entries $\mathcal{N} \sim\left(0, \sigma^{2}\right)$. Fix $\gamma>0$ and let us denote $\varepsilon:=(N+1)(1+\gamma) \sigma$. Since $\|\eta\|_{\ell_{2}}^{2}$ has a $\chi^{2}$ distribution with $(N+1)^{2}$ degrees of freedom, we have (see a comment to Lemma 1 in Section 4.1 in [32])

$$
\operatorname{Prob}\left(\|\eta\|_{\ell_{2}}>\varepsilon\right) \leq e^{-\frac{1}{2}(N+1)^{2} \gamma^{2}}
$$

Therefore,

$$
\left\|F_{N} f-y\right\|_{\ell_{2}}=\|\eta\|_{\ell_{2}} \leq \varepsilon
$$

with probability of at least $1-e^{-\frac{1}{2}(N+1)^{2} \gamma^{2}}$. The rest of the proof is identical to the proof of Theorem II.3.

\section{NUMERICAL EXPERIMENTS}

This section is devoted to extensive numerical experiments, examining both accuracy and complexity of Algorithm 1 The experiments were conducted in Matlab using CVX [25], which is the standard modeling system for convex optimization. The Matlab code is available on [1].

The signals were generated in the following two stages:

- Random locations on the sphere were drawn uniformly, sequentially added to the signal's support, while maintaining the separation condition of Definition II.1. In the non-negative case, the support was determined by $N$ random locations (no separation is needed).

- Once the support was determined, the amplitudes were drawn randomly from an iid normal distribution with standard deviation of $S D=10$. In the non-negative case, the amplitudes were drawn from a uniform distribution on the interval $[0,10]$.

The first experiment aims to estimate the separation constant $\nu$ from Definition II.1. For each values of $\nu, 20$ simulations were conducted and the error in the support localization was calculated. As can be seen in Figure 2, starting from $\nu=2 \pi$ 


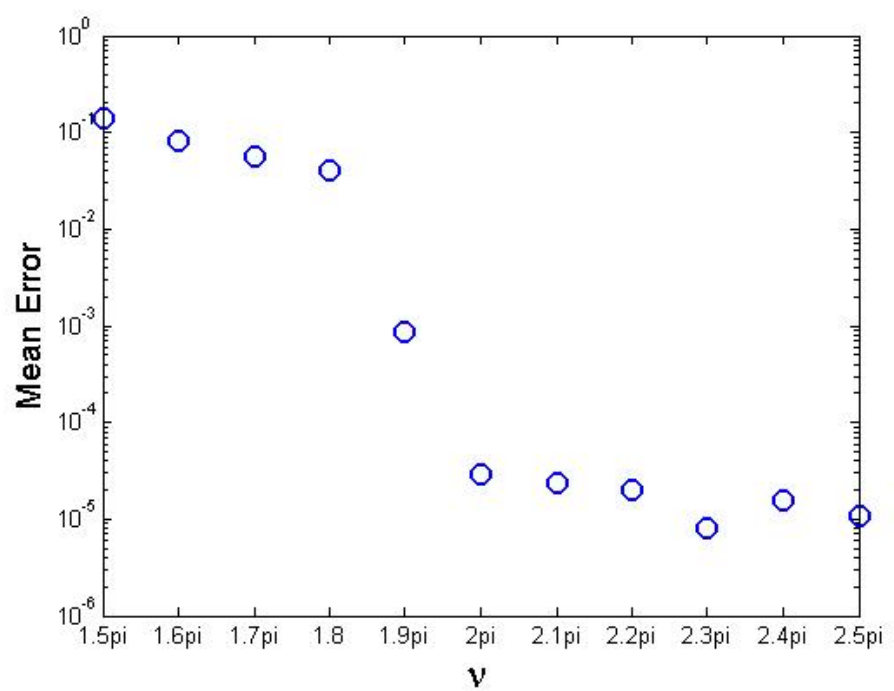

Fig. 2: The mean recoery error (in logarithmic scale) as a function of $\nu$ over 20 simulations. To be clear, by error we merely mean the distance on the sphere between the true and the estimated support.

the localization error is negligible. The result suggests that there exists a sharp phase transition for the relationship between the recovery error and $\nu$ (see [39] for the analysis of this phenomenon for signals defined on the circle). In the presence of noise, we found that increasing the separation constant to $\nu=2.5 \pi$ improves the results significantly.

An example to the performance of the algorithm is presented in Figure 3 Figure 3 a presents the low resolution measurements $P_{N} f:=F_{N}^{*} F_{N} f$ for $N=10$, and the recovered signal is presented in Figure $3 \mathrm{~b}$. We note that the recovered signal is identical to any visible accuracy to the original signal. As mentioned in Algorithm 1, the support is determined as the roots of the polynomials $1 \pm q(\xi)=1 \pm F_{N}^{*} \alpha(\xi)$, where $\alpha$ is the solution of the semi-definite program (III.8). Figure 4 presents $q(\xi)$ for the example of Figure 3

The roots of $1 \pm q(\xi)$ were located as follows. The sphere was divided into small cubes, and the minimum of the function at each cube was calculated using standard optimization tools. The minimum points with values below $10^{-5}$ were considered as roots. This technique exploits the fact that the signal's support is well separated. Finer segmentation of the sphere results in a better localization in the cost of computation time.

This experiment was conducted 10 times for $N=5,8,10$. Table I shows the error in estimating the support locations. Figure 5 shows an exact recovery of a clustered non-negative signal. As aforementioned, the separation condition is not

\footnotetext{
${ }^{2}$ The signal is presented on a grid for visualization purpose only.
} 


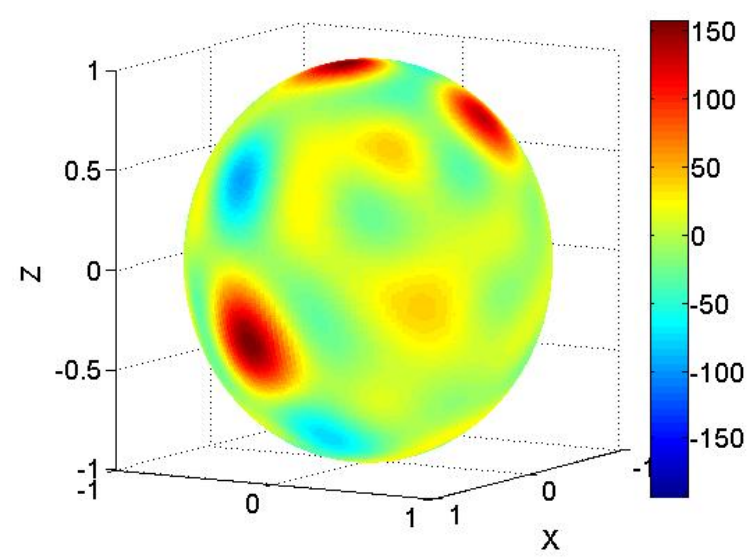

(a) The low resolution measurements $P_{N} f$, for $N=10$.

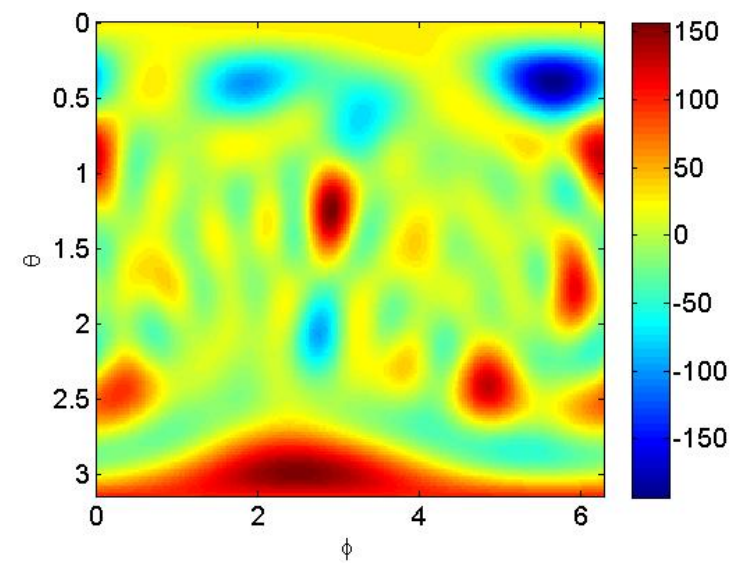

(c) The low resolution measurements $P_{N} f$, presented on a plane.

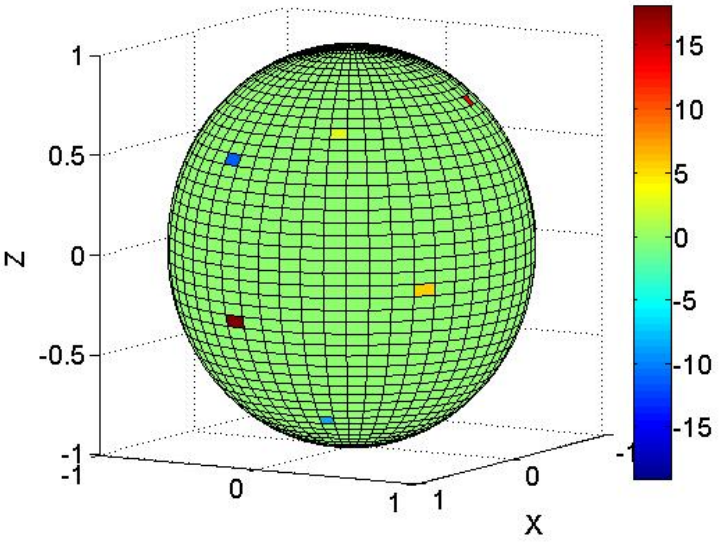

(b) The recovered signal $f$, for $N=10$.

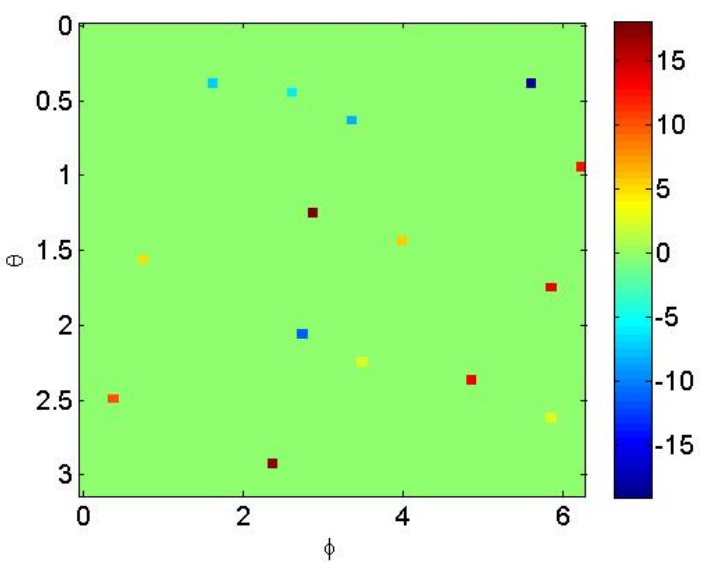

(d) The recovered signal $f$, presented on a plane.

Fig. 3: Super-resolution on the sphere using the Algorithm 1 for $N=10$. The signal is presented on a grid for visualization only.

necessary in this case.

In the discrete setting, both Algorithm 1 and $\ell_{1}$ minimization recover the signal exactly in a noise-free environment. In order to compare the algorithms, we applied both of them in the discrete setting, using the grid

$$
\mathbb{S}_{L}^{2}:=\left\{\left(\phi_{q}, \theta_{p}\right)=\left(2 \pi \frac{q}{L}, \pi \frac{p}{L}\right):(q, p) \subset[0,1, \ldots, L-1] \times[0,1, \ldots, L-1]\right\} .
$$




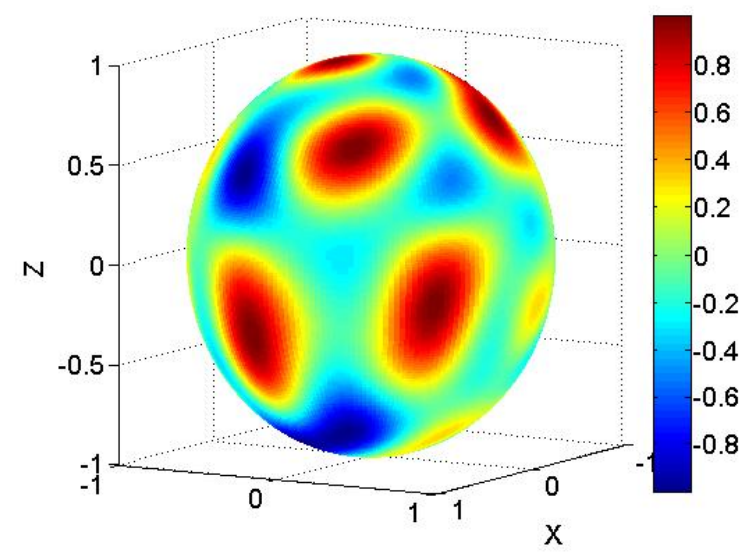

(a) The function $q(\xi)=F_{N}^{*} \alpha(\xi)$.

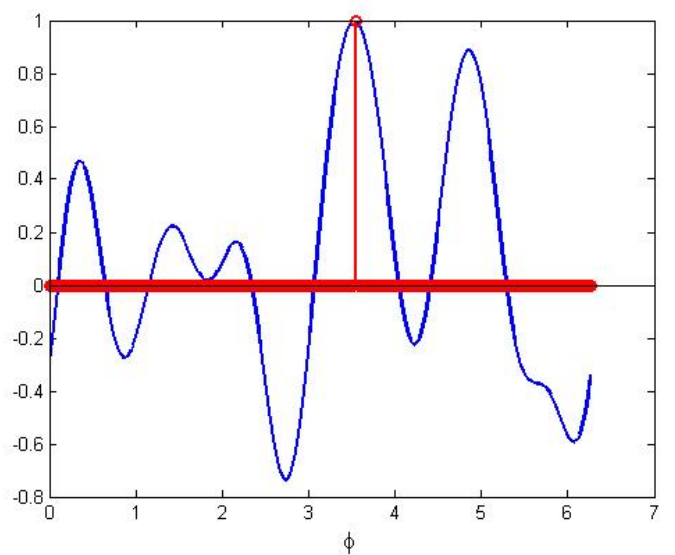

(b) A single line of $q(\xi)$ for a constant $\theta=2.257$ (blue) verses the appropriate values of $\operatorname{sign}(f)$ (red).

Fig. 4: The function $q(\xi)=F_{N}^{*} \alpha(\xi)$ for the example presented in Figure 3

\begin{tabular}{|c|c|c|c|}
\hline $\mathrm{N}$ & 5 & 8 & 10 \\
\hline \hline Average error & $8.1267 \times 10^{-5}$ & $8.1826 \times 10^{-5}$ & $9.0404 \times 10^{-5}$ \\
\hline Max error & $2.163 \times 10^{-4}$ & $1.9 \times 10^{-3}$ & $3.3 \times 10^{-3}$ \\
\hline
\end{tabular}

TABLE I: The localization error of Algorithm 1 for $N=5,8,10$. For each value of $N$, the experiment was conducted 10 times.

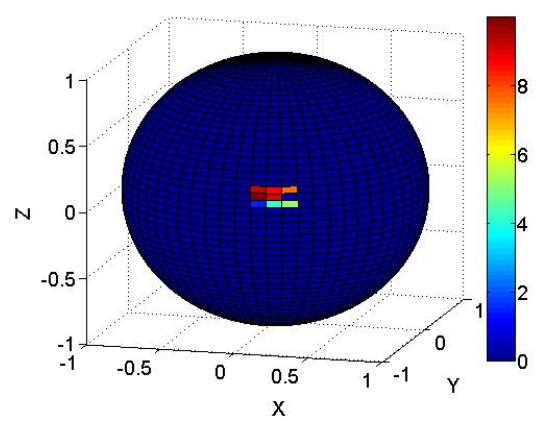

(a) The original signal $f$ with 9 non-zero values.

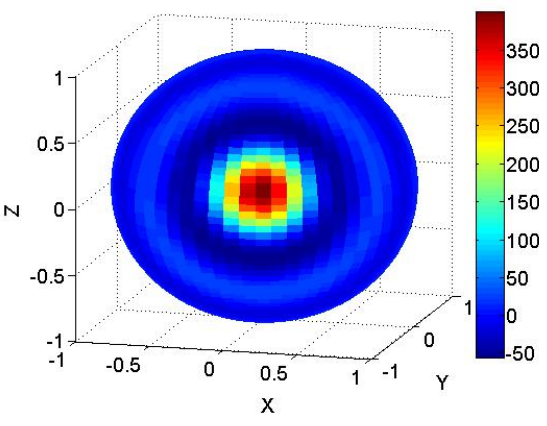

(b) The signal projection $P_{N} f$ for $N=9$.

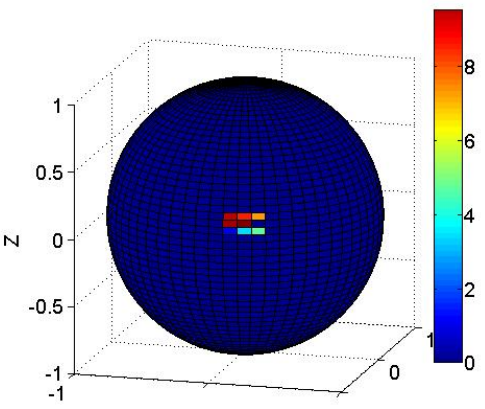

(c) The recovered signal.

Fig. 5: Recovery of a clustered non-negative signal. The signals are presented on a grid for convenient visualization. 


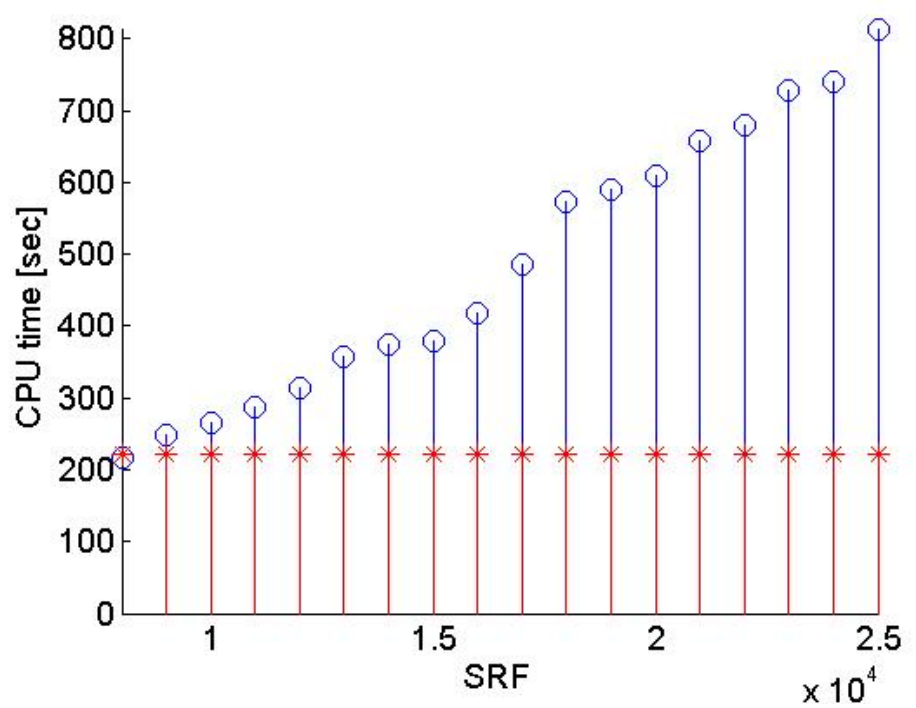

Fig. 6: CPU time of $\ell_{1}$ minimization and Algorithm 1 in the discrete setting with $N=5$. The red stars present the average computation time of Algorithm 1 over 100 experiments, and the blue circles present the average computation time (over 10 experiments) of the $\ell_{1}$ minimization as a function of the SRF.

Recall that the complexity of Algorithm 1 depends solely on $N$, while the complexity of the $\ell_{1}$ minimization depends on both $N$ and $L$. Therefore, finer grid results in a longer computation time. Figure 6 shows the computation time of $\ell_{1}$ minimization, as function of the SRF, compared with the average computation time of Algorithm 1 The $\ell_{1}$ minimization was solved using CVX [25]. As can be seen, the computation time of $\ell_{1}$ minimization grows (approximately) linearly with the SRF. In the last section we discuss some ideas how to speed up our algorithm.

In the noisy setting, we considered an additive noise with iid entries $\mathcal{N} \sim\left(0, \sigma^{2}\right)$. Our experiments show that although Theorem II.3 holds for recovery by $\ell_{1}$ minimization [II.4], Algorithm 1 is also robust to noise. In Figure 7 , we show the recovery error of Algorithm 1 with $\varepsilon=(1+N)(1+\gamma) \sigma$ for various of $\sigma$ and SRF values in the discrete setting. As can be seen, the error degrades moderately as $\sigma$ and SRF increase.

\section{THE NECESSITY OF THE SEPARATION CONDITION}

In [8], we established that the separation condition is a sufficient condition for exact recovery of a signal on a sphere from its projection onto $V_{N}$. Nonetheless, without separation the recovery task is ill-posed. Figure 8 shows an example for Algorithm 1 failure for clustered signals. The experiment was conducted with minimal separation of $\frac{2.5 \pi}{N^{1.5}}$, and $N=10$. 


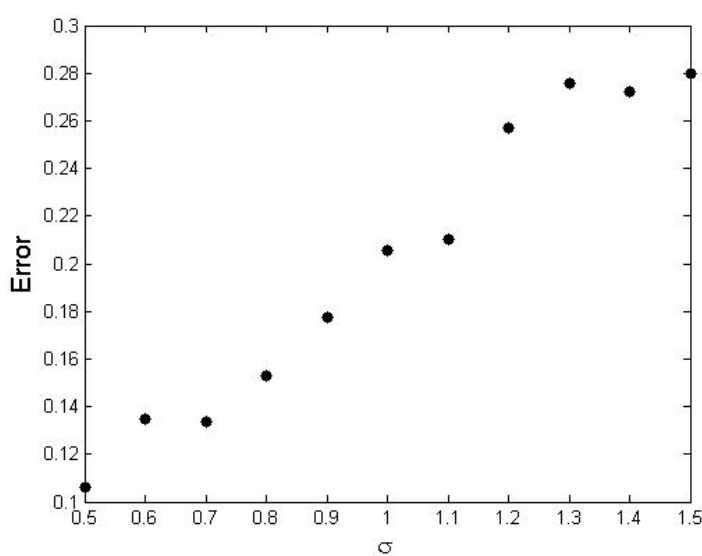

(a) The recovery error as a function of the noise standard deviation, for $S R F=10$.

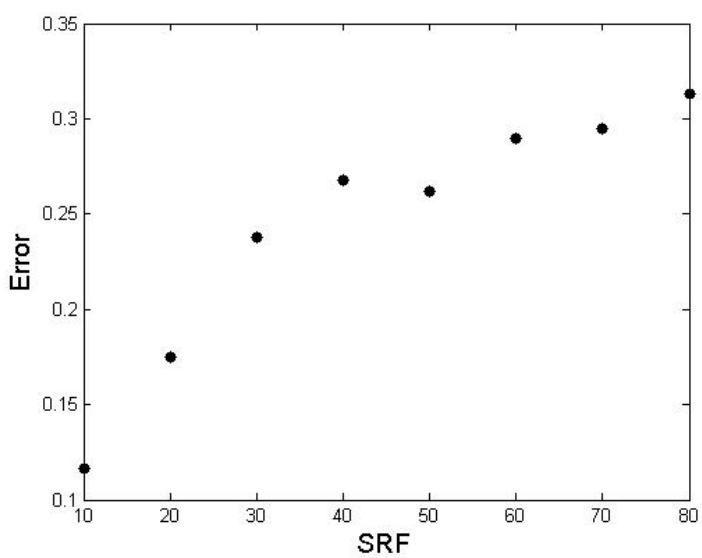

(b) The recovery error as a function of the SRF, for $\sigma=0.5$.

Fig. 7: For each value of $\sigma$ and SRF, 10 experiments were conducted using Algorithm 1 with $N=8, \gamma=1$, and $\varepsilon=(1+N)(1+\gamma) \sigma$. The figure presents the average recovery error. By error, we merely mean the distance on the sphere between the true and the estimated supports.

The points are scattered on $1 / N$ of the sphere, so the total number of locations is similar to the experiment presented in Figure 3

The recovery failure of the clustered signal can be explained by the analysis in [14], where the authors showed that clustered signals cannot be recovered by any method from their low frequency coefficients in the presence of minuscule noise level. They used prolate spheroidal sequences [49], and showed that asymptotically, even for small $S R F$ values, there will always exist an irretrievable signal. Furthermore, as the $S R F$ increases most of the information in a clustered signal is lost by the low-pass operation.

The formulation III.7 reveals that spherical harmonics expansion in $\mathbb{S}^{2}$ is a unique combination of bivariate trigonometric polynomials. Hence, the aforementioned conclusions hold for the spherical harmonic case as well, and we conclude that super-resolution on the sphere is ill-posed without a minimum separation condition.

To make this argument clear, we give here a simple example. Consider a signal of the form

$$
f=\delta_{\xi}-\delta_{\xi_{\epsilon}},
$$




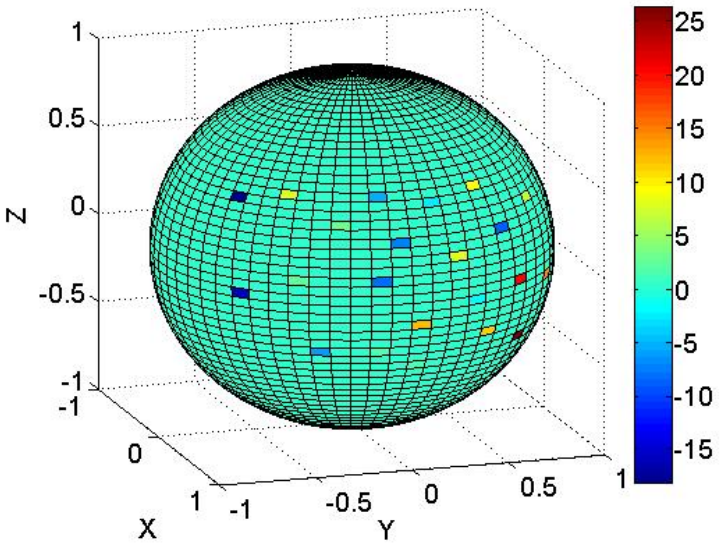

(a) Clustered signal with separation of $\frac{2.5 \pi}{N^{1.5}}$.

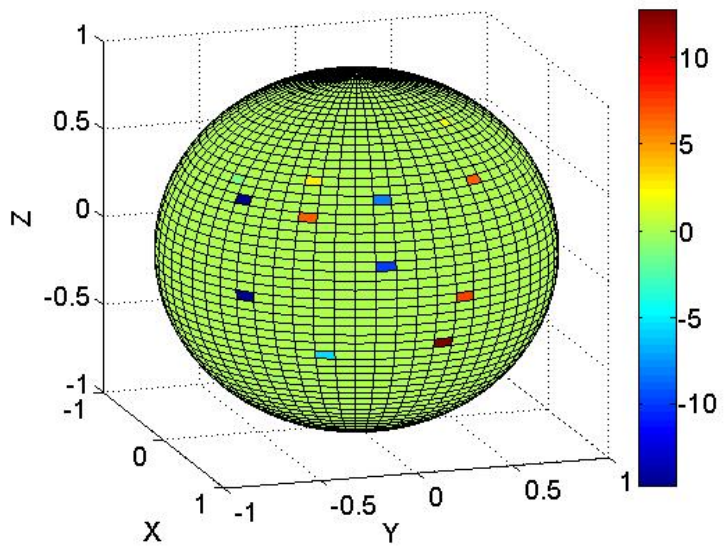

(b) The recovered signal.

Fig. 8: Unsuccessful recovery through Algorithm 1 of a clustered signal, $N=10$.

for some locations $\xi:=(\theta, \phi), \xi_{\epsilon}:=\left(\phi_{\epsilon}, \theta_{\epsilon}\right)$, and $d\left(\xi, \xi_{\epsilon}\right)=\epsilon$. In this case, the measurements are given by

$$
\begin{aligned}
y_{n, k} & =\bar{Y}_{n, k}(\xi)-\bar{Y}_{n, k}(\xi+\epsilon) \\
& =A_{n, k}\left[e^{-j k \phi} P_{n, k}(\cos \theta)-e^{-j k \phi_{\epsilon}} P_{n, k}\left(\cos \theta_{\epsilon}\right)\right] \\
& =A_{n, k} \sum_{\ell=-n}^{n} \beta_{n, k, l}\left[e^{-j k \phi} e^{-j \ell \theta}-e^{-j k \phi_{\epsilon}} e^{-j \ell \theta_{\epsilon}}\right] .
\end{aligned}
$$

When the spikes are close, i.e. $\epsilon \rightarrow 0$, we get $\left|y_{n, k}\right| \rightarrow 0$ for any $(n, k)$. As a conclusion, if the spikes are sufficiently close, all the measured information will be completely drowned in a small noise level.

\section{CONCLUSions And Future WORK}

In a previous paper, we have established that a signal of the form I.3 on the sphere can be recovered precisely from its projection onto spherical harmonics of degree $N$ using TV minimization, as long as the distance between the spikes is inversely proportional to $N$. In this paper, we suggested to recast the infinite dimensional TV minimization as a semidefinite program with $\mathcal{O}\left(N^{4}\right)$ variables. We showed that Algorithm 1 recovers the signal with high precision and that its complexity does not depend on the resolution. We strongly believe that this result holds in higher dimensions and for complex Dirac ensembles. Indeed, significant parts of the proof can be easily generalized to any dimension and to complex signals. However, there are certain technical challenges which we hope to overcome in future work. 
Furthermore, we showed that in the discrete configuration, recovery by $\ell_{1}$ minimization is robust to noise, and the recovery error is proportional to the noise standard deviation and $S R F^{2}$. We showed experimentally that similar estimation holds for Algorithm 1 as well.

Our algorithm can be improved in two directions. As aforementioned, the semi-definite program was implemented using CVX on Matlab. CVX was designed as a convenient tool for convex optimization, however, it does not purport to be efficient. In order to speed the computation time, one needs to design its own solver, which is beyond the scope of this paper. Another direction is designing an algorithm, dedicated for root finding on the sphere.

Finally, this paper is part of an ongoing research, suggesting to solve infinite dimensional convex optimization problems using a finite semi-definite programs. Up to now, these method were applied to projections onto trigonometric [11], [13], [14], [16], [53], [54], [56] and algebraic polynomial spaces [9], [19]. This work showed that it can be applied to the sphere as well. An interesting question is whether this approach can be applied to recover a signal lying on complicate geometries from their projection onto harmonic polynomials. We leave this for a future research.

\section{ACKNOWLEDGEMENT}

We thank the referees for their valuable comments that have significantly improved this paper.

\section{REFERENCES}

[1] http://www.shaidekel.com.

[2] Simon R Arridge. Optical tomography in medical imaging. Inverse problems, 15(2):R41, 1999.

[3] Kendall Atkinson and Weimin Han. Spherical harmonics and approximations on the unit sphere: an introduction, volume 2044. Springer, 2012.

[4] Pascal Audet. Directional wavelet analysis on the sphere: Application to gravity and topography of the terrestrial planets. Journal of Geophysical Research: Planets (1991-2012), 116(E1), 2011.

[5] Jean-Marc Azais, Yohann De Castro, and Fabrice Gamboa. Spike detection from inaccurate samplings. Applied and Computational Harmonic Analysis, 2014.

[6] Ilan Ben Hagai, Filippo Maria Fazi, and Boaz Rafaely. Generalized sampling expansion for functions on the sphere. Signal Processing, IEEE Transactions on, 60(11):5870-5879, 2012.

[7] Tamir Bendory, Avinoam Bar-Zion, Shai Dekel, Arie Feuer, and Dan Adam. Robust and localized recovery of stream of pulses with application to ultrasound imaging. in preparation.

[8] Tamir Bendory, Shai Dekel, and Arie Feuer. Exact recovery of dirac ensembles from the projection onto spaces of spherical harmonics. Constructive Approximation, to appear, 2014.

[9] Tamir Bendory, Shai Dekel, and Arie Feuer. Exact recovery of non-uniform splines from the projection onto spaces of algebraic polynomials. Journal of Approximation Theory, 182(0):7 - 17, 2014.

[10] Tamir Bendory, Shai Dekel, and Arie Feuer. Robust recovery of streams of pulses using convex optimization. submitted, 2014.

[11] Badri Narayan Bhaskar and Benjamin Recht. Atomic norm denoising with applications to line spectral estimation. In Communication, Control, and Computing (Allerton), 2011 49th Annual Allerton Conference on, pages 261-268. IEEE, 2011. 
[12] Nicolas Burq, Semyon Dyatlov, Rachel Ward, and Maciej Zworski. Weighted eigenfunction estimates with applications to compressed sensing. SIAM Journal on Mathematical Analysis, 44(5):3481-3501, 2012.

[13] Emmanuel J Candès and Carlos Fernandez-Granda. Super-resolution from noisy data. Journal of Fourier Analysis and Applications, 19(6):12291254, 2013.

[14] Emmanuel J Candès and Carlos Fernandez-Granda. Towards a mathematical theory of super-resolution. Communications on Pure and Applied Mathematics, 67(6):906-956, 2014.

[15] Emmanuel J Candès, Justin Romberg, and Terence Tao. Robust uncertainty principles: Exact signal reconstruction from highly incomplete frequency information. Information Theory, IEEE Transactions on, 52(2):489-509, 2006.

[16] Yuejie Chi and Yuxin Chen. Compressive recovery of 2-d off-grid frequencies. In Proceedings of Asilomar Conference on Signals, Systems, and Computers, 2013.

[17] Claude Cohen-Tannoudji, Bernard Diu, Frank Laloe, and Bernard Dui. Quantum Mechanics (2 vol. set). Wiley-Interscience, October 2006.

[18] Yohann De Castro and Fabrice Gamboa. Exact reconstruction using beurling minimal extrapolation. Journal of Mathematical Analysis and applications, 395(1):336-354, 2012.

[19] Yohann De Castro and Guillaume Mijoule. Non-uniform spline recovery from small degree polynomial approximation. arXiv preprint arXiv:1402.5662, 2014.

[20] S. Deslauriers-Gauthier and P. Marziliano. Sampling signals with a finite rate of innovation on the sphere. Signal Processing, IEEE Transactions on, 61(18):4552-4561, Sept 2013.

[21] Samuel Deslauriers-Gauthier and Pina Marziliano. Spherical finite rate of innovation theory for the recovery of fiber orientations. In Engineering in Medicine and Biology Society (EMBC), 2012 Annual International Conference of the IEEE, pages 2294-2297. IEEE, 2012.

[22] David L Donoho. Compressed sensing. Information Theory, IEEE Transactions on, 52(4):1289-1306, 2006.

[23] Bogdan Dumitrescu. Positive trigonometric polynomials and signal processing applications. Springer, 2007.

[24] Carlos Fernandez-Granda. Support detection in super-resolution. arXiv preprint arXiv:1302.3921, 2013.

[25] Michael Grant and Stephen Boyd. CVX: Matlab software for disciplined convex programming, version 2.1, March 2014.

[26] Y. Hua and T.K. Sarkar. Matrix pencil method for estimating parameters of exponentially damped/undamped sinusoids in noise. Acoustics, Speech and Signal Processing, IEEE Transactions on, 38(5):814-824, 1990.

[27] Daniel P Jarrett, Emanuël AP Habets, and Patrick A Naylor. 3d source localization in the spherical harmonic domain using a pseudointensity vector. In Proc. European Signal Processing Conf.(EUSIPCO), Aalborg, Denmark, pages 442-446, 2010.

[28] Zubair Khalid, Rodney A Kennedy, and Jason D McEwen. An optimal-dimensionality sampling scheme on the sphere for fast spherical harmonic transforms. arXiv preprint arXiv:1403.4661, 2014.

[29] Zubair Khalid, Parastoo Sadeghi, Rodney A Kennedy, and Salman Durrani. Spatially varying spectral filtering of signals on the unit sphere. Signal Processing, IEEE Transactions on, 61(3):530-544, 2013.

[30] SM Klosko and CA Wagner. Spherical harmonic representation of the gravity field from dynamic satellite data. Planetary and Space Science, 30(1):5-28, 1982.

[31] Eiichiro Komatsu, KM Smith, J Dunkley, CL Bennett, B Gold, G Hinshaw, N Jarosik, D Larson, MR Nolta, L Page, et al. Seven-year wilkinson microwave anisotropy probe (wmap) observations: cosmological interpretation. The Astrophysical Journal Supplement Series, 192(2):18, 2011.

[32] Béatrice Laurent and Pascal Massart. Adaptive estimation of a quadratic functional by model selection. Annals of Statistics, pages 1302-1338, 2000.

[33] Boris Leistedt and Jason D McEwen. Exact wavelets on the ball. Signal Processing, IEEE Transactions on, 60(12):6257-6269, 2012.

[34] Thomas Murray MacRobert and Ian Naismith Sneddon. Spherical harmonics: an elementary treatise on harmonic functions with applications, volume 3. Pergamon Press, 1967. 
[35] Jason D McEwen, Gilles Puy, J-P Thiran, Pierre Vandergheynst, Dimitri Van De Ville, and Yves Wiaux. Sparse image reconstruction on the sphere: implications of a new sampling theorem. Image Processing, IEEE Transactions on, 22(6):2275-2285, 2013.

[36] Jason D McEwen and Yves Wiaux. A novel sampling theorem on the sphere. Signal Processing, IEEE Transactions on, 59(12):5876-5887, 2011.

[37] Jens Meyer. Beamforming for a circular microphone array mounted on spherically shaped objects. The Journal of the Acoustical Society of America, 109(1):185-193, 2001.

[38] Jens Meyer and Tony Agnello. Spherical microphone array for spatial sound recording. In Audio Engineering Society Convention 115. Audio Engineering Society, 2003.

[39] Ankur Moitra. The threshold for super-resolution via extremal functions. arXiv preprint arXiv:1408.1681, 2014.

[40] Tony S Pollock, Thushara D Abhayapala, and Rodney A Kennedy. Introducing space into mimo capacity calculations. Telecommunication Systems, 24(2-4):415-436, 2003.

[41] Boaz Rafaely. Plane-wave decomposition of the sound field on a sphere by spherical convolution. The Journal of the Acoustical Society of America, 116(4):2149-2157, 2004.

[42] Boaz Rafaely. Analysis and design of spherical microphone arrays. Speech and Audio Processing, IEEE Transactions on, 13(1):135-143, 2005.

[43] Robert Alexander Rankin. The closest packing of spherical caps in n dimensions. In Proceedings of the glasgow mathematical association, volume 2, pages 139-144. Cambridge Univ Press, 1955.

[44] Holger Rauhut and Rachel Ward. Sparse recovery for spherical harmonic expansions. arXiv preprint arXiv:1102.4097, 2011.

[45] Richard Roy and Thomas Kailath. Esprit-estimation of signal parameters via rotational invariance techniques. Acoustics, Speech and Signal Processing, IEEE Transactions on, 37(7):984-995, 1989.

[46] Walter Rudin. Real and complex analysis (3rd). New York: McGraw-Hill Inc, 1986.

[47] Ralph O Schmidt. Multiple emitter location and signal parameter estimation. Antennas and Propagation, IEEE Transactions on, 34(3):276-280, 1986.

[48] Frederik J Simons, FA Dahlen, and Mark A Wieczorek. Spatiospectral concentration on a sphere. SIAM review, 48(3):504-536, 2006.

[49] David Slepian. Prolate spheroidal wave functions, fourier analysis, and uncertainty v: The discrete case. Bell System Technical Journal, 57(5):13711430, 1978 .

[50] Peter-Pike Sloan. Stupid spherical harmonics (sh) tricks. In Game Developers Conference, volume 9, 2008.

[51] Petre Stoica and Randolph L Moses. Spectral analysis of signals. Pearson/Prentice Hall Upper Saddle River, NJ, 2005.

[52] Katsuyuki Taguchi, Gengsheng L Zeng, and Grant T Gullberg. Cone-beam image reconstruction using spherical harmonics. Physics in medicine and biology, 46(6):N127, 2001.

[53] Gongguo Tang, Badri Narayan Bhaskar, and Benjamin Recht. Near minimax line spectral estimation. In Information Sciences and Systems (CISS), 2013 47th Annual Conference on, pages 1-6. IEEE, 2013.

[54] Gongguo Tang, Badri Narayan Bhaskar, Parikshit Shah, and Benjamin Recht. Compressive sensing off the grid. In Communication, Control, and Computing (Allerton), 2012 50th Annual Allerton Conference on, pages 778-785. IEEE, 2012.

[55] J Tournier, Fernando Calamante, David G Gadian, and Alan Connelly. Direct estimation of the fiber orientation density function from diffusionweighted mri data using spherical deconvolution. NeuroImage, 23(3):1176-1185, 2004.

[56] Weiyu Xu, Jian-Feng Cai, Kumar Vijay Mishra, Myung Cho, and Anton Kruger. Precise semidefinite programming formulation of atomic norm minimization for recovering d-dimensional (d>=2) off-the-grid frequencies. In Information Theory and Applications Workshop (ITA), 2014, pages 1-4. IEEE, 2014. 